\title{
Melemium Hydrogensulfate H3C6N7(NH2)3(HSO4)3 - the First Triple Protonation of Melem
}

\begin{tabular}{|c|c|}
\hline Journal: & Zeitschrift für Anorganische und Allgemeine Chemie \\
\hline Manuscript ID: & zaac. 201000246.R1 \\
\hline Wiley - Manuscript type: & Article \\
\hline $\begin{array}{r}\text { Date Submitted by the } \\
\text { Author: }\end{array}$ & 28-Jul-2010 \\
\hline Complete List of Authors: & $\begin{array}{l}\text { Sattler, Andreas; LMU Munich, Chemistry } \\
\text { Schnick, Wolfgang; LMU Munich, Chemistry }\end{array}$ \\
\hline Keywords: & Heptazine, Tri-s-triazine, Crystal structure \\
\hline
\end{tabular}

\section{SCHOLARONE $^{\text {TH }}$ Manuscripts}


Keywords: Heptazine; Tri-s-triazine; Crystal Structure.

\title{
Melemium Hydrogensulfate $\mathrm{H}_{3} \mathrm{C}_{6} \mathrm{~N}_{7}\left(\mathrm{NH}_{2}\right)_{3}\left(\mathrm{HSO}_{4}\right)_{3}$ - the First Triple Protonation of Melem
}

\author{
Andreas Sattler and Wolfgang Schnick*
}

In this contribution an overview of accessible melemium sulfates depending on the concentration of sulfuric acid is given. Two additional melemium sulfates were identified this way.

\footnotetext{
* Prof. Dr. W. Schnick Department Chemie Lehrstuhl für Anorganische Festkörperchemie Ludwig-Maximilians-Universität München Butenandtstraße 5 - 13 (D) D-81377 München, Germany Fax: +49-(0)89-2180-77440 E-mail: wolfgang.schnick@uni-muenchen.de

Supporting information for this article is available on the WWW under http://dx.doi.org/10.1002/zaac.201000xxx or from the author
}

\section{Introduction}

The $s$-heptazine (tri-s-triazine, $\left[\mathrm{C}_{6} \mathrm{~N}_{7}\right]$ ) core (cf. Scheme 1) constitutes a fundamental structural motif for carbon nitride materials. It is of considerable interest as a building block discussed for graphitic carbon nitride $\left(\mathrm{g}-\mathrm{C}_{3} \mathrm{~N}_{4}\right)$ [1-3]. The heptazine core is also known as cyameluric nucleus, a name coined by Pauling [4] referring to cyameluric acid $\mathrm{H}_{3} \mathrm{C}_{6} \mathrm{~N}_{7} \mathrm{O}_{3}$ [5]. Although much of the current interest concerning heptazines initially originated from the "harder than diamond" considerations based on calculations for certain modifications of $\mathrm{C}_{3} \mathrm{~N}_{4}[6,7]$ additional properties (catalysis being a prominent example $[3 \mathrm{a}, 8,9]$ ) have recently broadened the focus of carbon nitride chemistry.

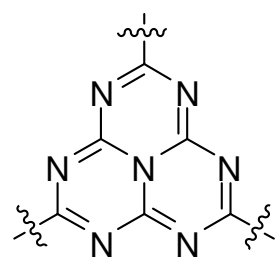

Typically, many heptazine-containing compounds are synthesized based on the thermal condensation of melamine (2,4,6-triamino-s-triazine, $\left.\mathrm{C}_{3} \mathrm{~N}_{3}\left(\mathrm{NH}_{2}\right)_{3}\right)$. The molecular compound melem (2,6,10-triamino-s-heptazine, $\mathrm{C}_{6} \mathrm{~N}_{7}\left(\mathrm{NH}_{2}\right)_{3}$, cf. Scheme 2) is a prominent example of a product obtained accordingly. Though known for a considerable period of time [10], melem has not been comprehensively characterized until very recently. Melem [11], some of its salts [12] and its molecular adducts with melamine [13] were thus discussed in prior investigations. We have found that melem can easily adopt different protonation grades (single and double protonation) in several compounds. So far, triple protonation of melem has not been observed in a crystalline compound.<smiles>Nc1nc(N)nc(N)n1</smiles><smiles>NC1=NC2=NC(N)=NC3=NC(N)=NC(=N1)N23</smiles>

\section{A}

Scheme 2. Structure of melamine $\mathrm{C}_{3} \mathrm{~N}_{3}\left(\mathrm{NH}_{2}\right)_{3}$ (A) and melem $\mathrm{C}_{6} \mathrm{~N}_{7}\left(\mathrm{NH}_{2}\right)_{3}$ (B).

In this contribution we report on the first example of a triple protonated $\mathrm{H}_{3} \mathrm{C}_{6} \mathrm{~N}_{7}\left(\mathrm{NH}_{2}\right)_{3}{ }^{3+}$ ion in the melemium hydrogensulfate salt $\mathrm{H}_{3} \mathrm{C}_{6} \mathrm{~N}_{7}\left(\mathrm{NH}_{2}\right)_{3}\left(\mathrm{HSO}_{4}\right)_{3}$. The compound was prepared by reaction of melem with $70 \%$ sulfuric acid. We have investigated the structure and properties of this novel salt. Additionally, we present studies concerning the

Scheme 1. The cyameluric nucleus $\left[\mathrm{C}_{6} \mathrm{~N}_{7}\right]$. 
Table 1. Existence ranges of different melemium salts in relation to the concentration of sulfuric acid.

\begin{tabular}{|c|c|c|c|c|c|}
\hline \multicolumn{6}{|c|}{ Compounds observed as part of this work } \\
\hline conc. $\left(\mathrm{H}_{2} \mathrm{SO}_{4}\right) / \mathrm{wt} \%$ & $0-0.04$ & $7-40$ & $44-48$ & $53-58$ & $>69$ \\
\hline molarity $\left(\mathrm{H}_{2} \mathrm{SO}_{4}\right) / \mathrm{mol} \mathrm{l}^{-1}[\mathrm{a}]$ & $0-0.4$ & $0.78-4.9$ & $5.6-6.3$ & $7.2-8$ & $>10$ \\
\hline molality $\left(\mathrm{H}_{2} \mathrm{SO}_{4}\right) / \mathrm{mol} \mathrm{kg}^{-1}$ & $0-0.41$ & $0.82-6.7$ & $8.1-9.4$ & $12-14$ & $>23$ \\
\hline observed compound [b] & melem hydrate & $\mathrm{H}_{2} \mathrm{C}_{6} \mathrm{~N}_{7}\left(\mathrm{NH}_{2}\right)_{3}\left(\mathrm{SO}_{4}\right) \cdot 2 \mathrm{H}_{2} \mathrm{O}$ & new salt $\mathrm{A}$ & new salt B & $\mathrm{H}_{3} \mathrm{C}_{6} \mathrm{~N}_{7}\left(\mathrm{NH}_{2}\right)_{3}\left(\mathrm{HSO}_{4}\right)_{3}$ \\
\hline
\end{tabular}

[a] calculated neglecting excess volumes; [b] identified by XRD methods.

formation of different melemium sulfate salts depending on the respective concentration of sulfuric acid.

\section{Results and Discussion}

Different salts were observed upon subjecting melem to aqueous sulfuric acid solutions of various concentrations. This finding is in line with the observations made for the perchloric and methylsulfonic acid salts of melem, where a variety of salts has been found, as well [12]. While the concentration of the acid is a crucial factor, the amount of melem dissolved or suspended in the acid is only of minor importance. Values between 1 and $10 \mathrm{mg}$ per $\mathrm{mL}$ of solution are suitable for lower sulfuric acid concentrations, while higher concentrations allow more melem to be used. As the concentration of $\mathrm{H}_{2} \mathrm{SO}_{4}$ rises the solubility of melem drastically increases. Melem also becomes prone to decomposition under these conditions. Complete decomposition requires boiling and rather long reaction times. This decomposition ultimately produces $\left(\mathrm{NH}_{4}\right)_{2} \mathrm{SO}_{4}$ and $\mathrm{CO}_{2}$, however, intermediary occurrence of other compounds is to be expected.

We have characterized the salt $\mathrm{H}_{2} \mathrm{C}_{6} \mathrm{~N}_{7}\left(\mathrm{NH}_{2}\right)_{3}\left(\mathrm{SO}_{4}\right)$. $2 \mathrm{H}_{2} \mathrm{O}$ [12a] in our previous investigations. The comprehensive study of the formation of melemium sulfate salts revealed a total of three new compounds (cf. Table 1). Starting from the concentrations known to produce $\mathrm{H}_{2} \mathrm{C}_{6} \mathrm{~N}_{7}\left(\mathrm{NH}_{2}\right)_{3}\left(\mathrm{SO}_{4}\right) \cdot 2 \mathrm{H}_{2} \mathrm{O}$ [12a] we have identified new salts obtainable at higher concentrations of sulfuric acid. There, however, seem to be no additional salts that can be yielded at lower sulfuric acid concentrations. Instead the formation of melem hydrate [14] was observed for very dilute solutions. The list in Table 1 does not claim to be exhaustive since not all possible concentrations were investigated. Two of the three new salts discovered could unfortunately not be structurally studied as we have not succeeded in growing suitable single crystals. The third compound was yielded well-crystalline and could be investigated by single-crystal XRD. The formula was found to be $\mathrm{H}_{3} \mathrm{C}_{6} \mathrm{~N}_{7}\left(\mathrm{NH}_{2}\right)_{3}\left(\mathrm{HSO}_{4}\right)_{3}$, hence the compound is a triple-protonated melemium hydrogensulfate.

One might assume the acidity of $\mathrm{H}_{3} \mathrm{C}_{6} \mathrm{~N}_{7}\left(\mathrm{NH}_{2}\right)_{3}{ }^{3+}$ being higher than the one of $\mathrm{HSO}_{4}{ }^{-}$as no $\mathrm{SO}_{4}{ }^{2-}$ is found in the compound. The $\mathrm{pK}_{s}$ value for $\mathrm{HSO}_{4}{ }^{-}$is given as 1.92 [15], the $\mathrm{K}_{b}$ values published for melem are $\mathrm{K} b_{1}=10^{-12}, \mathrm{~K} b_{2}=$ $6 \cdot 10^{-14}$, and $\mathrm{K} b_{3}=10^{-16}$ [16]. These values are supportive to this assumption, however, quite problematic, since solidstate and solution acidities usually differ and values are only applicable for very dilute solutions. Overall activity coefficients of $\mathrm{H}_{2} \mathrm{SO}_{4}$ in aqueous solution show a minimum for moderately concentrated solutions and rise by more than a magnitude $\left(0.119\right.$ for $\mathrm{c}=1 \mathrm{~mol} \mathrm{~kg}^{-1}$ to 1.701 for $\mathrm{c}=$
$20 \mathrm{~mol} \mathrm{~kg}^{-1}$ [17]) going from concentrations found to produce $\mathrm{H}_{2} \mathrm{C}_{6} \mathrm{~N}_{7}\left(\mathrm{NH}_{2}\right)_{3}\left(\mathrm{SO}_{4}\right) \cdot 2 \mathrm{H}_{2} \mathrm{O}$ to the ones yielding $\mathrm{H}_{3} \mathrm{C}_{6} \mathrm{~N}_{7}\left(\mathrm{NH}_{2}\right)_{3}\left(\mathrm{HSO}_{4}\right)_{3}$ (cf. Table 1). The importance of $\mathrm{SO}_{4}^{2-}$ ions also diminishes in concentrated solutions. Solutions like those used for preparing compound A contain less than $1 \% \mathrm{SO}_{4}{ }^{2-}$, for the higher concentrated acids sulfate concentration is negligible [18]. The existence of the two other salts $\mathrm{A}$ and $\mathrm{B}$ suggests that triple protonation of melem might be possible at lower $\mathrm{H}_{2} \mathrm{SO}_{4}$ concentrations, as well and the compounds most likely incorporate the $\mathrm{HSO}_{4}{ }^{-}$ion.

$\mathrm{H}_{3} \mathrm{C}_{6} \mathrm{~N}_{7}\left(\mathrm{NH}_{2}\right)_{3}\left(\mathrm{HSO}_{4}\right)_{3}$ is an extremely hygroscopic compound. Crystals of the title compound decay within split-seconds when subjected to moist air, thus the compound needs to be handled in an inert gas atmosphere. By reaction with moisture the compound is transformed into another melemium salt (a colorless solid) that, judging from powder XRD, is not identical with either one of the salts accessible from solution (cf. Table 1). After over-night exposure of $\mathrm{H}_{3} \mathrm{C}_{6} \mathrm{~N}_{7}\left(\mathrm{NH}_{2}\right)_{3}\left(\mathrm{HSO}_{4}\right)_{3}$ to air, elemental analysis data (cf. supporting information) suggests the uptake of 3-4 mole equivalents of water. Taking this properties into account, $\mathrm{H}_{3} \mathrm{C}_{6} \mathrm{~N}_{7}\left(\mathrm{NH}_{2}\right)_{3}\left(\mathrm{HSO}_{4}\right)_{3}$ might be used as desiccant or moisture sensor.

Table 2. Crystallographic data and details of the structure refinement for $\mathrm{H}_{3} \mathrm{C}_{6} \mathrm{~N}_{7}\left(\mathrm{NH}_{2}\right)_{3}\left(\mathrm{HSO}_{4}\right)_{3}$.

\begin{tabular}{|c|c|}
\hline formula & $\mathrm{H}_{3} \mathrm{C}_{6} \mathrm{~N}_{7}\left(\mathrm{NH}_{2}\right)_{3}\left(\mathrm{HSO}_{4}\right)_{3}$ \\
\hline formula weight $/ \mathrm{g} \mathrm{mol}^{-1}$ & 512.44 \\
\hline crystal system & monoclinic \\
\hline space group & $P 2_{1} / n$ (no. 14$)$ \\
\hline \multirow[t]{3}{*}{ lattice parameters / $\AA{ }^{\circ}$} & $a=10.277(2)$ \\
\hline & $b=14.921(3)$ \\
\hline & $c=11.771(2)$ \\
\hline volume / $\AA^{3}$ & $1781.5(6)$ \\
\hline $\mathrm{Z}$ & 4 \\
\hline diffractometer & Stoe IPDS \\
\hline radiation, monochromator & Mo-K ${ }_{\alpha}(\lambda=71.073 \mathrm{pm})$, graphite \\
\hline temperature / K & 293 \\
\hline structure solution & SHELXS [20] (direct methods) \\
\hline structure refinement & SHELXL [20] (full-matrix least-squares on $\mathrm{F}^{2}$ ) \\
\hline corrections applied & Lorentz, polarization \\
\hline absorption coefficient / $\mathrm{mm}^{-1}$ & 0.507 \\
\hline calculated density $/ \mathrm{g} \mathrm{cm}^{-3}$ & 1.911 \\
\hline data / restraints / parameters & $4087 / 0 / 328$ \\
\hline \multirow[t]{4}{*}{$R$-indices } & $R_{1}=0.0614$ all data \\
\hline & $R_{1}=0.0371 \mathrm{~F}_{\mathrm{O}}^{2}>2 \sigma\left(\mathrm{F}_{\mathrm{O}}^{2}\right)$ (2894 reflections) \\
\hline & $w R_{2}=0.0904$ all data \\
\hline & $w R_{2}=0.0851 \mathrm{~F}_{\mathrm{O}}^{2}>2 \sigma\left(\mathrm{F}_{\mathrm{O}}^{2}\right)$ \\
\hline GooF & 0.947 \\
\hline \multirow[t]{2}{*}{ weighting scheme } & $\mathrm{w}^{-1}=\sigma^{2}\left(\mathrm{~F}_{\mathrm{o}}^{2}\right)+(0.0510 \mathrm{P})^{2}$ \\
\hline & where $\mathrm{P}=\left(\mathrm{F}_{\mathrm{o}}^{2}+2 \mathrm{~F}_{\mathrm{c}}^{2}\right) / 3$ \\
\hline largest peak / deepest hole / e $\AA^{-3}$ & $0.367 /-0.393$ \\
\hline
\end{tabular}

As mentioned above, the structure of the title compound was solved by single-crystal XRD (cf. Table 2). 
$\mathrm{H}_{3} \mathrm{C}_{6} \mathrm{~N}_{7}\left(\mathrm{NH}_{2}\right)_{3}\left(\mathrm{HSO}_{4}\right)_{3}$ crystallizes in the monoclinic space group $P 2_{1} / n$ with 4 formula units per unit cell. The asymmetric unit comprises one $\mathrm{H}_{3} \mathrm{C}_{6} \mathrm{~N}_{7}\left(\mathrm{NH}_{2}\right)_{3}{ }^{3+}$ ion and three hydrogensulfate ions. No solvate molecules are integrated into the compound.

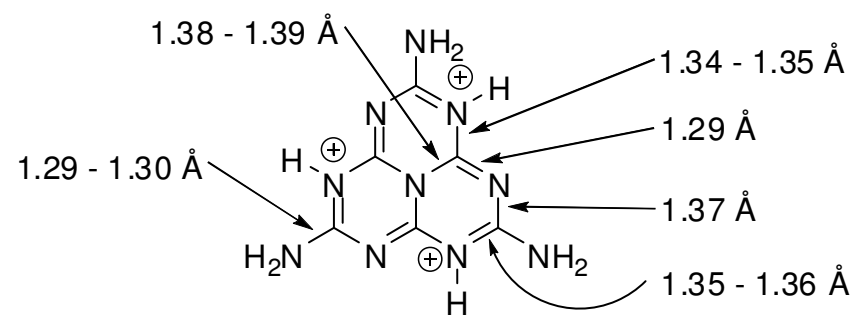

Scheme 3. Bond lengths observed for the melemium ion found in $\mathrm{H}_{3} \mathrm{C}_{6} \mathrm{~N}_{7}\left(\mathrm{NH}_{2}\right)_{3}\left(\mathrm{HSO}_{4}\right)_{3}$.

The $\mathrm{H}_{3} \mathrm{C}_{6} \mathrm{~N}_{7}\left(\mathrm{NH}_{2}\right)_{3}{ }^{3+}$ ions display three protonation sites at the nitrogen atoms of the cyameluric nucleus. As expected the protons avoid close proximity, resulting in an arrangement closely resembling $C_{3 h}$ symmetry (cf. Scheme 3, Figure 1). The bond lengths within the heptazine core are notably affected by protonation (cf. Table 3 ). This is in accordance with observations for other melemium salts [12]. Bonds are influenced in a way that can best be described by assuming a slight preference for the resonance structure presented in Scheme 3. The $\mathrm{C}-\mathrm{N}$ bonds to the amino groups are $2-3 \mathrm{pm}$ shorter than the respective bonds in melem [11b].

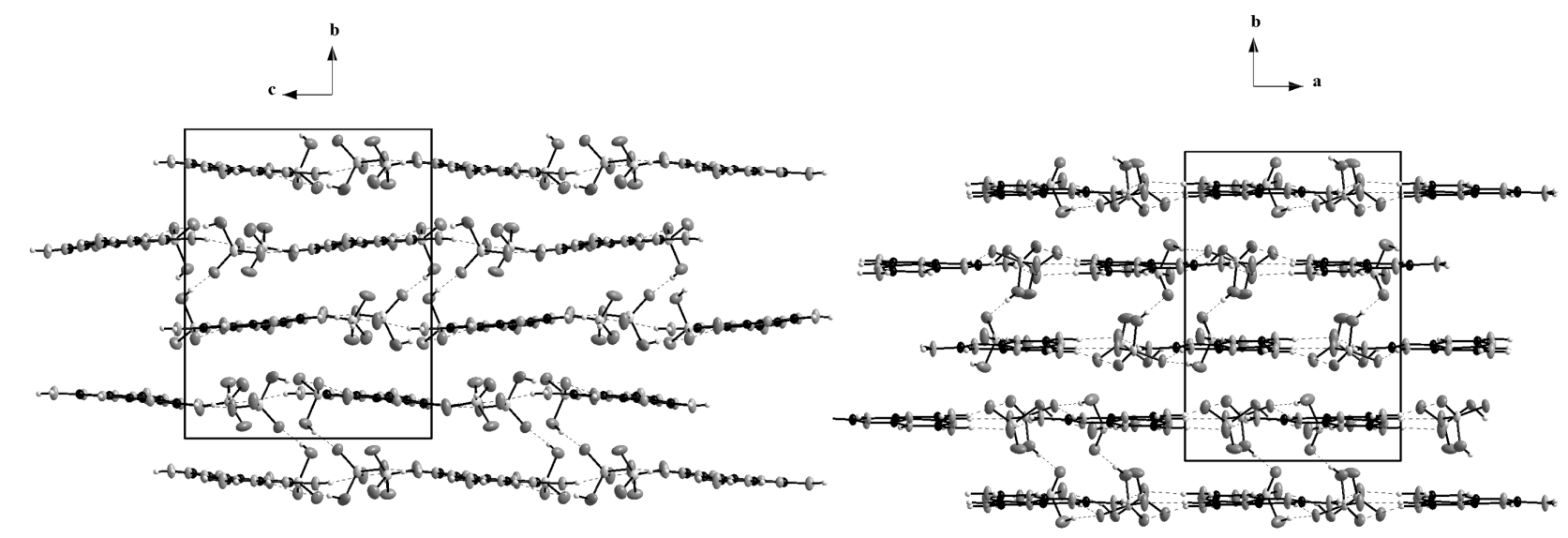

Figure 2. Layer-like arrangement of melemium ions in $\mathrm{H}_{3} \mathrm{C}_{6} \mathrm{~N}_{7}\left(\mathrm{NH}_{2}\right)_{3}\left(\mathrm{HSO}_{4}\right)_{3}$. Views along $a$ (left) and $c$ (right). Ellipsoids drawn at the $50 \%$ probability level. 


\begin{tabular}{|c|c|c|c|c|c|c|c|c|c|}
\hline \multicolumn{4}{|c|}{ Distances } & \multicolumn{6}{|c|}{ Angles } \\
\hline C1-N7 & $1.294(3)$ & O1-S1 & $1.4629(18)$ & N7-C1-N2 & $120.6(2)$ & $\mathrm{C} 1-\mathrm{N} 2-\mathrm{C} 5$ & $122.38(19)$ & $\mathrm{S} 1-\mathrm{O} 3-\mathrm{H} 10$ & 101(3) \\
\hline $\mathrm{C} 1-\mathrm{N} 2$ & $1.341(3)$ & $\mathrm{O} 2-\mathrm{S} 1$ & $1.425(2)$ & N7-C1-N1 & $123.48(19)$ & C1-N2-H3 & $118(2)$ & S2-O6-H12 & $107(3)$ \\
\hline $\mathrm{C} 2-\mathrm{N} 3$ & $1.290(3)$ & $\mathrm{O} 3-\mathrm{H} 10$ & $0.93(5)$ & $\mathrm{N} 3-\mathrm{C} 2-\mathrm{N} 4$ & $121.02(19)$ & $\mathrm{C} 2-\mathrm{N} 3-\mathrm{C} 5$ & $117.02(18)$ & $\mathrm{O} 2-\mathrm{S} 1-\mathrm{O} 4$ & $115.87(14)$ \\
\hline $\mathrm{C} 2-\mathrm{N} 4$ & $1.350(3)$ & O4-S1 & $1.439(2)$ & N3-C2-N1 & $123.84(19)$ & C2-N4-C6 & $122.18(18)$ & $\mathrm{O} 2-\mathrm{S} 1-\mathrm{O} 1$ & $111.72(13)$ \\
\hline $\mathrm{C} 2-\mathrm{N} 1$ & 1.391(3) & $\mathrm{O} 5-\mathrm{S} 2$ & $1.4322(17)$ & $\mathrm{N} 4-\mathrm{C} 2-\mathrm{N} 1$ & $115.14(18)$ & $\mathrm{C} 2-\mathrm{N} 4-\mathrm{H} 9$ & $115(2)$ & O4-S1-O1 & $111.45(11)$ \\
\hline C3-N6 & $1.342(3)$ & O6-H12 & $0.81(4)$ & $\mathrm{N} 5-\mathrm{C} 3-\mathrm{N} 1$ & $123.56(19)$ & C3-N5-C6 & $117.14(18)$ & $\mathrm{O} 4-\mathrm{S} 1-\mathrm{O} 3$ & $104.29(12)$ \\
\hline $\mathrm{C} 3-\mathrm{N} 1$ & $1.384(3)$ & O7-S2 & $1.4555(18)$ & N6-C3-N1 & $115.51(18)$ & C3-N6-C4 & $122.54(18)$ & $\mathrm{O} 1-\mathrm{S} 1-\mathrm{O} 3$ & $106.36(11)$ \\
\hline C4-N10 & $1.299(3)$ & O8-S2 & $1.4673(17)$ & N10-C4-N7 & $118.9(2)$ & C3-N6-H6 & $116.9(19)$ & $\mathrm{O} 5-\mathrm{S} 2-\mathrm{O} 7$ & $113.24(11)$ \\
\hline C4-N7 & $1.356(3)$ & O9-S3 & $1.4640(18)$ & N10-C4-N6 & $119.9(2)$ & C4-N6-H6 & $120.4(19)$ & $\mathrm{O} 5-\mathrm{S} 2-\mathrm{O} 8$ & $113.44(11)$ \\
\hline C4-N6 & $1.367(3)$ & O10-S3 & $1.548(2)$ & N7-C4-N6 & $121.11(19)$ & C1-N7-C4 & $117.08(19)$ & $\mathrm{O} 7-\mathrm{S} 2-\mathrm{O} 8$ & $109.77(10)$ \\
\hline C5-N8 & $1.291(3)$ & O10-H11 & $0.83(4)$ & $\mathrm{N} 8-\mathrm{C} 5-\mathrm{N} 3$ & $119.6(2)$ & $\mathrm{C} 5-\mathrm{N} 8-\mathrm{H} 1$ & $121.5(18)$ & $\mathrm{O} 5-\mathrm{S} 2-\mathrm{O} 6$ & $105.00(11)$ \\
\hline $\mathrm{C} 5-\mathrm{N} 3$ & $1.353(3)$ & O11-S3 & $1.4450(19)$ & $\mathrm{N} 8-\mathrm{C} 5-\mathrm{N} 2$ & $119.1(2)$ & $\mathrm{C} 5-\mathrm{N} 8-\mathrm{H} 2$ & $122(2)$ & O7-S2-O6 & $107.56(13)$ \\
\hline C6-N9 & $1.302(3)$ & N8-H1 & $0.92(3)$ & N9-C6-N5 & $118.4(2)$ & C6-N9-H7 & $119.0(17)$ & O11-S3-O12 & $114.24(11)$ \\
\hline C6-N5 & $1.350(3)$ & $\mathrm{N} 8-\mathrm{H} 2$ & $0.77(3)$ & N9-C6-N4 & $119.9(2)$ & C6-N9-H8 & $120(2)$ & O11-S3-O9 & $112.04(12)$ \\
\hline C6-N4 & $1.369(3)$ & N9-H7 & $0.93(3)$ & N5-C6-N4 & $121.72(19)$ & H7-N9-H8 & $120(3)$ & O12-S3-O9 & $111.43(11)$ \\
\hline $\mathrm{N} 2-\mathrm{H} 3$ & $0.81(3)$ & N9-H8 & $0.80(3)$ & C3-N1-C1 & $119.76(17)$ & C4-N10-H4 & $121(2)$ & O11-S3-O10 & $107.63(11)$ \\
\hline N4-H9 & $0.81(3)$ & N10-H4 & $0.91(3)$ & C3-N1-C2 & $119.98(17)$ & C4-N10-H5 & $122.4(19)$ & O12-S3-O10 & $105.18(13)$ \\
\hline N6-H6 & $0.86(3)$ & $\mathrm{N} 10-\mathrm{H} 5$ & $0.84(3)$ & C1-N1-C2 & $119.28(17)$ & $\mathrm{H} 4-\mathrm{N} 10-\mathrm{H} 5$ & $116(3)$ & O9-S3-O10 & $105.64(13)$ \\
\hline
\end{tabular}

supporting this assumption. Typical vibrations for $\mathrm{N}-\mathrm{C}$ and $\mathrm{S}-\mathrm{O}$ bonds were also found.

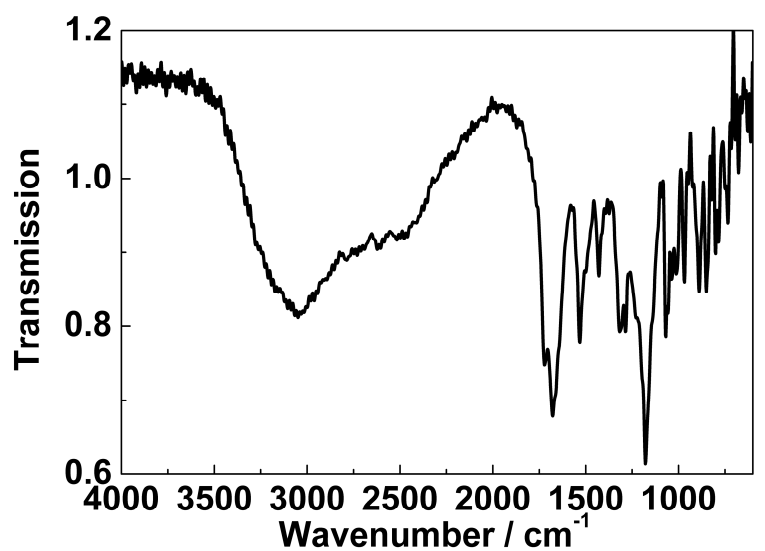

Figure 3. IR Spectrum $(\mathrm{KBr})$ of $\mathrm{H}_{3} \mathrm{C}_{6} \mathrm{~N}_{7}\left(\mathrm{NH}_{2}\right)_{3}\left(\mathrm{HSO}_{4}\right)_{3}$.

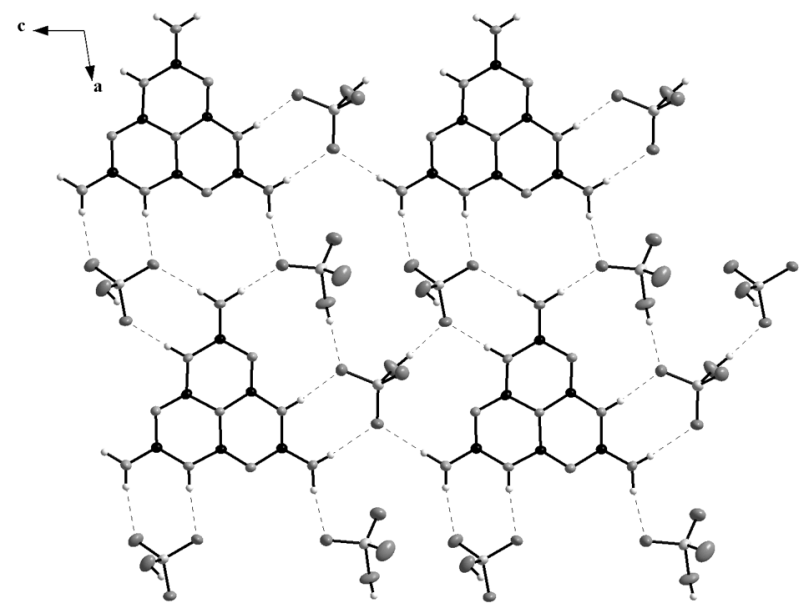

Figure 4. A H-bonded layer of melemium ions found in $\mathrm{H}_{3} \mathrm{C}_{6} \mathrm{~N}_{7}\left(\mathrm{NH}_{2}\right)_{3}\left(\mathrm{HSO}_{4}\right)_{3}$. View along $b$. Ellipsoids drawn at the $50 \%$ probability level.

Similarly to $\mathrm{HC}_{6} \mathrm{~N}_{7}\left(\mathrm{NH}_{2}\right)_{3} \mathrm{H}_{2} \mathrm{C}_{6} \mathrm{~N}_{7}\left(\mathrm{NH}_{2}\right)_{3}\left(\mathrm{SO}_{3} \mathrm{Me}\right)_{3} \cdot \mathrm{H}_{2} \mathrm{O}$ and $\mathrm{H}_{2} \mathrm{C}_{6} \mathrm{~N}_{7}\left(\mathrm{NH}_{2}\right)_{3}\left(\mathrm{SO}_{3} \mathrm{Me}\right)$, two melemium methylsulfonate salts which we described recently [12c], $\mathrm{H}_{3} \mathrm{C}_{6} \mathrm{~N}_{7}\left(\mathrm{NH}_{2}\right)_{3}\left(\mathrm{HSO}_{4}\right)_{3}$ also features short distances between $\mathrm{S}=\mathrm{O}$ moieties and carbon or nitrogen atoms within the cyameluric nucleus. Especially the distances of the oxygen atom $\mathrm{O} 2$ to the atoms $\mathrm{N} 1(2.67 \AA)$ and $\mathrm{C} 2(2.69 \AA)$ are remarkably short (cf. Figure 1 for assignment). However, comparable distances are not unreasonable and have been reported in the literature [19]. Some examples even display notably shorter atomic distances. $\mathrm{H}_{3} \mathrm{C}_{6} \mathrm{~N}_{7}\left(\mathrm{NH}_{2}\right)_{3}\left(\mathrm{HSO}_{4}\right)_{3}$ shows the shortest such contacts yet found for melemium salts. This might be due to the higher positive charge of the $\mathrm{H}_{3} \mathrm{C}_{6} \mathrm{~N}_{7}\left(\mathrm{NH}_{2}\right)_{3}{ }_{3}^{3+}$ ion making interactions with negatively polarized oxygen atoms of the sulfate ions more favorable 
than has been the case in salts with lower protonation of melem.

$\mathrm{H}_{3} \mathrm{C}_{6} \mathrm{~N}_{7}\left(\mathrm{NH}_{2}\right)_{3}\left(\mathrm{HSO}_{4}\right)_{3}$ shows high thermal stability (cf. Figure 5). No thermal events were observed below $200{ }^{\circ} \mathrm{C}$ The endothermic event at $210^{\circ} \mathrm{C}$ followed by a gradual weight loss can probably be explained by melting under decomposition. Another endothermic effect associated with a high weight loss is observed onsetting at $370{ }^{\circ} \mathrm{C}$. Total decomposition is observed above $600{ }^{\circ} \mathrm{C}$.

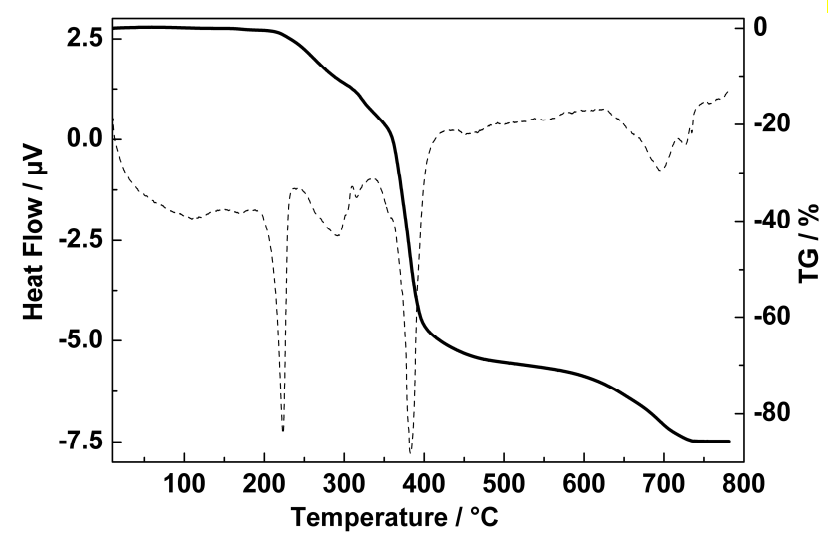

Figure 5. DTA/TG diagram of $\mathrm{H}_{3} \mathrm{C}_{6} \mathrm{~N}_{7}\left(\mathrm{NH}_{2}\right)_{3}\left(\mathrm{HSO}_{4}\right)_{3}$.

\section{Conclusions}

The structure of $\mathrm{H}_{3} \mathrm{C}_{6} \mathrm{~N}_{7}\left(\mathrm{NH}_{2}\right)_{3}\left(\mathrm{HSO}_{4}\right)_{3}$, the first tripleprotonated melemium salt, was reported. The compound was yielded by crystallization from ca. $70 \%$ aqueous sulfuric acid. The $\mathrm{H}_{3} \mathrm{C}_{6} \mathrm{~N}_{7}\left(\mathrm{NH}_{2}\right)_{3}{ }^{3+}$ ion was found to exhibit $C_{3 v}$ symmetry. The molecular structure of melem is characteristically affected by the protonation of the cyameluric nucleus. We have also investigated the stability of other melemium salts with respect to the concentration of sulfuric acid, showing the existence of two other salts the structure of which we, however, have yet not been able to elucidate.

\section{Experimental Section}

Single-crystal XRD: The crystal data of $\mathrm{H}_{3} \mathrm{C}_{6} \mathrm{~N}_{7}\left(\mathrm{NH}_{2}\right)_{3}\left(\mathrm{HSO}_{4}\right)_{3}$ was measured on a Stoe IPDS diffractometer. The measurement was conducted at a temperature of $293 \mathrm{~K}$. The crystal structure was solved by direct methods (SHELXS-97) and refined against $\mathrm{F}^{2}$ on all data by full-matrix-least-squares (SHELXL-97) [20]. Crystallographic data is summarized in Table 2. Selected distances and angles are presented in Table 3. Further details on the crystal structure can be obtained free of charge on application to Cambridge Crystallographic Data Centre (CCDC 768285), 12 Union Road, Cambridge CB2 1EZ (UK) (fax: +(44) 1223-336033); e-mail (fileserv@ ccdc.cam.ac.uk).

PXRD diagrams were recorded on a Huber G670 Guinier Imaging Plate or a Stoe STADI P diffractometer. Measurements were conducted using monochromatic $\mathrm{Cu}-\mathrm{K}_{\alpha 1}$ radiation at room temperature

Thermal Analysis was performed using a Setaram TG-92 simultaneous thermal analyzer equipped with a protected (i.e. alumina covered) DTA/TG rod (P-type thermocouple, maximum operating temperature $\left.=1600{ }^{\circ} \mathrm{C}\right) .22 .5 \mathrm{mg} \mathrm{H}_{3} \mathrm{C}_{6} \mathrm{~N}_{7}\left(\mathrm{NH}_{2}\right)_{3}\left(\mathrm{HSO}_{4}\right)_{3}$ were heated to $800{ }^{\circ} \mathrm{C}$ in an atmosphere of streaming helium at a scanning rate of $10 \mathrm{~K} \mathrm{~min}^{-1}$ using a $100 \mu \mathrm{L}$ alumina crucible.

FTIR measurements were conducted on an IFS 66v/S FTIR spectrometer (Bruker). $\mathrm{KBr}$ pellets were prepared in an argon glove box.

Melem: Melem was yielded by condensation of melamine in accordance to literature procedures $[11,12 \mathrm{c}]$.

The title compound is very hygroscopic and rapidly decays when subjected to air. Isolation and all manipulations of $\mathrm{H}_{3} \mathrm{C}_{6} \mathrm{~N}_{7}\left(\mathrm{NH}_{2}\right)_{3}\left(\mathrm{HSO}_{4}\right)_{3}$ were thus carried out under dry argon using Schlenk techniques.

Melemium hydrogensulfate: Melem $(4.2 \mathrm{~g}, 19.3 \mathrm{mmol})$ was dissolved in a hot mixture of conc. $\mathrm{H}_{2} \mathrm{SO}_{4}(35 \mathrm{~mL})$ and water $(15 \mathrm{~mL})$. This needs to be conducted fast to avoid decomposition. The solution was stored at $4{ }^{\circ} \mathrm{C}$ over night. The colorless crystals were collected by filtration using a sintered glass frit washed twice with glacial acetic acid and dried at $10^{-3}$ mbar for at least $24 \mathrm{~h}$. $\mathrm{H}_{3} \mathrm{C}_{6} \mathrm{~N}_{7}\left(\mathrm{NH}_{2}\right)_{3}\left(\mathrm{HSO}_{4}\right)_{3}$ was yielded as a colorless solid $(3.89 \mathrm{~g}$, $7.6 \mathrm{mmol}, 39 \%)$.

FTIR $\left(\mathrm{KBr}, 25^{\circ} \mathrm{C}, \mathrm{cm}^{-1}\right) 3040 \mathrm{~s}$ vbr, $1721 \mathrm{~m}, 1678 \mathrm{~s}, 1531 \mathrm{~m}$, $1429 \mathrm{w}, 1317 \mathrm{~m}, 1285 \mathrm{~m}, 1178 \mathrm{~s}$ br, $1068 \mathrm{~m}, 1037 \mathrm{w}, 1012 \mathrm{w}$, $968 \mathrm{~m}, 889 \mathrm{~m}, 851 \mathrm{~m}, 801 \mathrm{w}, 784 \mathrm{w}, 734 \mathrm{w}, 588 \mathrm{~m}, 572 \mathrm{~s}$.

Crystals were selected in an argon glove box and measured in a sealed glass capillary.

Supporting information contains further experimental and analytical data for compounds A and B.

\section{Acknowledgments}

The authors would like to thank T. Miller, Department Chemie LMU München, for collecting the X-ray diffraction data. Financial support by the Deutsche Forschungsgemeinschaft DFG (project SCHN 377/12) as well as the Fonds der Chemischen Industrie (FCI) is gratefully acknowledged.

[1] E. Kroke, M. Schwarz, E. Horvath-Bordon, P. Kroll, B. Noll, A. D. Norman, New J. Chem. 2002, 26, 508.

[2] a) T. Komatsu, T. Nakamura, J. Mater. Chem. 2001, 11, 474; b) T. Komatsu, J. Mater. Chem. 2001, 11, 802.

[3] a) A. Thomas, A. Fischer, F. Goettmann, M. Antonietti, J.-O. Müller, R. Schlögl, J. M. Carlsson, J. Mater. Chem. 2008, 18, 4893; b) X. Wang, K. Maeda, A. Thomas, K. Takanabe, G. Xin, J. M. Carlsson, K. Domen, M. Antonietti, Nat. Mater. 2009, 8,76 .

[4] L. Pauling, J. H. Sturdivant, Proc. Natl. Acad. Sci. U.S.A. 1937, 23, 615.

[5] a) A. Sattler, W. Schnick, Z. Anorg. Allg. Chem. 2006, 632, 1518; b) A. Sattler, W. Schnick, Z. Anorg. Allg. Chem. 2006, 632, 531; c) N.E.A. El-Gamel, L. Seyferth, J. Wagner, H. Ehrenberg, M. Schwarz, J. Senker, E. Kroke, Chem. Eur. J. 2007, 13, 1158; d) E. Horvath-Bordon, E. Kroke, I. Svoboda, H. Fueß, R. Riedel, S. Neeraj, A. K. Cheetham, Dalton Trans. 2004, 22, 3900; e) J. Wagler, N. E. A. El-Gamel, E. Kroke, Z. Naturforsch. 2006, 61b, 975; f) W. Henneberg, Ann. Chem. Pharm. 1850, 73, 228.

[6] D. M. Teter, R. J. Hemley, Science 1996, 271, 53.

[7] a) A. Y. Liu, M. L. Cohen, Science 1989, 245, 841; b) A. Y. Liu, M. L. Cohen, Phys. Rev. B 1990, 41, 10727; c) A. Y. Liu, R. M. Wentzcovitch, Phys. Rev. B 1994, 50, 10362.

[8] a) F. Goettmann, A. Fischer, M. Antonietti, A. Thomas, Chem. Commun. 2006, 4530; b) F. Goettmann, A. Fischer, M. 
Antonietti, A. Thomas, New. J. Chem. 2007, 31, 1455; c) F. Goettmann, A. Thomas, M. Antonietti, Angew. Chem. 2007, 119, 2773; Angew. Chem. Int. Ed. 2007, 46, 2717.

[9] Y. Zhang, A. Thomas, M. Antonietti, X. Wang, J. Am. Chem. Soc. 2009, 131, 1384

[10] J. Liebig, Ann. Pharm. 1834, 10, 1.

[11] a) B. Jürgens, E. Irran, J. Senker, P. Kroll, H. Müller, W. Schnick, J. Am. Chem. Soc. 2003, 125, 10288; b) A. Sattler, W. Schnick, Z. Anorg. Allg. Chem. 2006, 632, 238.

[12] a) A. Sattler, L. Seyfarth, J. Senker, W. Schnick, Z. Anorg. Allg. Chem. 2005, 631, 2545; b) A. Sattler, W. Schnick, Z. Anorg. Allg. Chem. 2008, 634, 457; c) A. Sattler, S Schönberger, W. Schnick, Z. Anorg. Allg. Chem. 2010, 636, 476.

[13] A. Sattler, S. Pagano, M. Zeuner, A. Zurawski, D. Gunzelmann, J. Senker, K. Müller-Buschbaum, W. Schnick, Chem. Eur. J. 2009, 15, 13161.

[14] The respective hydrate of melem of the supposed formula $\mathrm{C}_{6} \mathrm{~N}_{7}\left(\mathrm{NH}_{2}\right)_{3} \cdot 1.5 \mathrm{H}_{2} \mathrm{O}$ was observed during our previous investigations: A. Sattler, Diploma Thesis, University of Munich (LMU), 2005.

[15] Handbook of Chemistry and Physics, (Editor in Chief R. C. Weast) The Chemical Rubber Co., Cleveland, Ohio, $49^{\text {th }}$ Edition, 1968, p. D91.

[16] A. I. Finkel'shtein, N. V. Spiridonova, Russ. Chem. Rev. 1964, 33, 400

[17] Handbook of Chemistry and Physics, (Editor in Chief D. R. Lide) CRC Press., Boca Raton London New York, $88^{\text {th }}$ Edition, 2007, p. 5-82.

[18] Gmelins Handbuch der Anorganischen Chemie, Schwefel, Verlag Chemie, Weinheim, $8^{\text {th }}$ Edition 1960, Teil B, pp.737.

[19] We have found some other compounds displaying short contacts between a $\mathrm{S}=\mathrm{O}$ oxygen atom and a $\mathrm{C}-\mathrm{N}$ bond. Examples are: CCDC 608134, CCDC 100391, CCDC 146348, CCDC 141245 and CSD 57371. Data was found using CCDC ConQuest v.1.11.

[20] G. M. Sheldrick, Acta Crystallogr. 2008, A64, 112. 
Supporting Information:

Results and Discussion

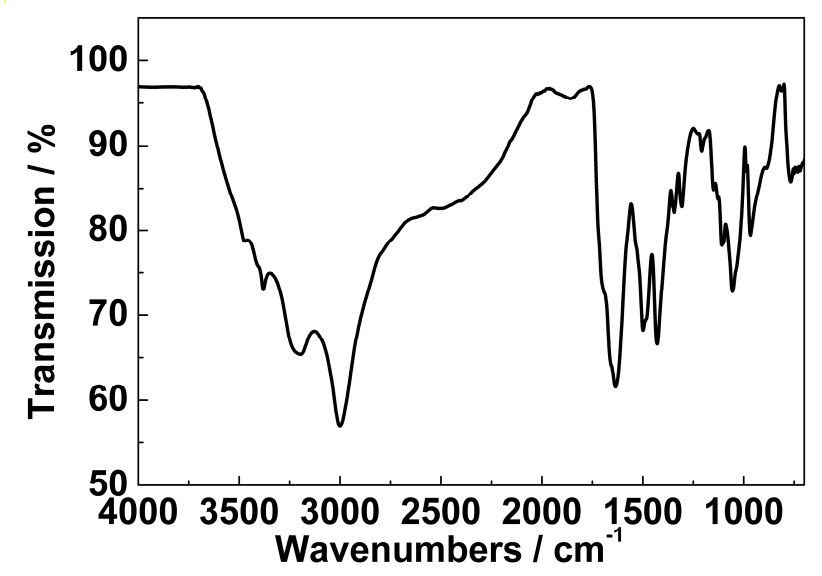

Figure S1. IR Spectrum (ATR, room temperature) of Compound A.

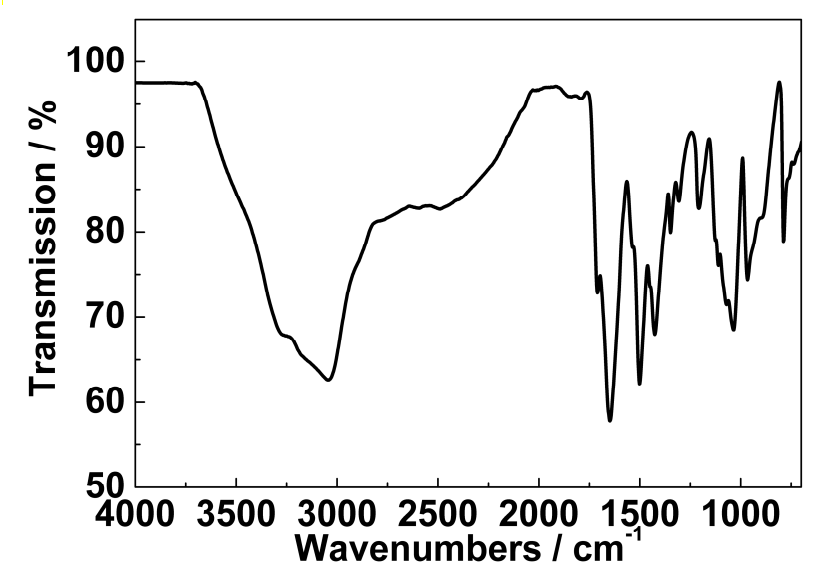

Figure S2. IR Spectrum (ATR, room temperature) of Compound B.

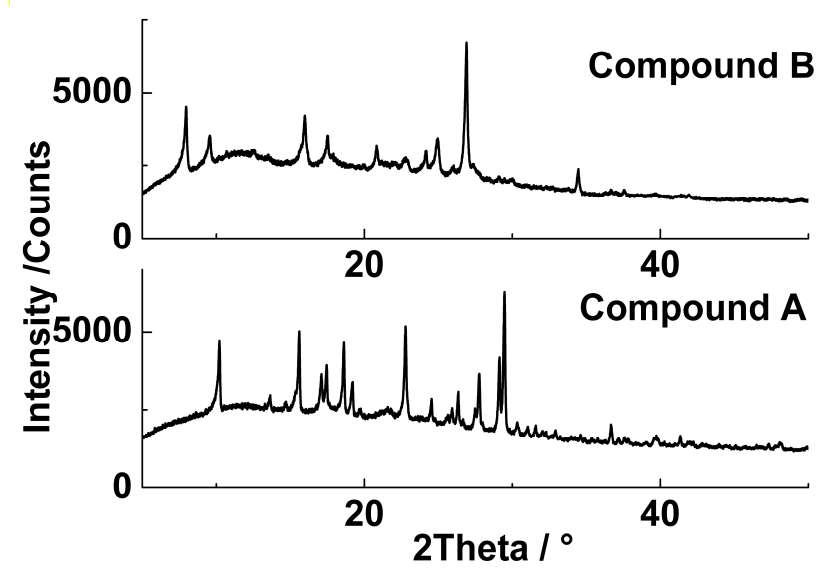

Figure $\mathrm{S} 3$. PXRD patterns $\left(\mathrm{Cu}_{\mathrm{k} \alpha 1}\right.$ Radiation) of compounds $\mathrm{A}$ and $\mathrm{B}$. 
Table S1. Elemental analysis for the decomposition product title compound after over-night exposure to air.

\begin{tabular}{lllll}
\hline & $\mathrm{C}$ & $\mathrm{N}$ & $\mathrm{H}$ & $\mathrm{S}$ \\
\hline found & 11.27 & 21.65 & 3.63 & 16.13 \\
calcd for $\mathrm{H}_{3} \mathrm{C}_{6} \mathrm{~N}_{7}\left(\mathrm{NH}_{2}\right)_{3}\left(\mathrm{HSO}_{4}\right)_{3}$ & 14.06 & 27.33 & 2.36 & 18.14 \\
calcd for $\mathrm{H}_{3} \mathrm{C}_{6} \mathrm{~N}_{7}\left(\mathrm{NH}_{2}\right)_{3}\left(\mathrm{HSO}_{4}\right)_{3}+3 \mathrm{H}_{2} \mathrm{O}$ & 11.96 & 23.25 & 3.68 & 15.97 \\
calcd for $\mathrm{H}_{3} \mathrm{C}_{6} \mathrm{~N}_{7}\left(\mathrm{NH}_{2}\right)_{3}\left(\mathrm{HSO}_{4}\right)_{3}+4 \mathrm{H}_{2} \mathrm{O}$ & 11.61 & 22.57 & 3.90 & 15.50 \\
\hline
\end{tabular}

\section{Experimental Section.}

Compound A: Melem Melem (1.00 g, $4.587 \mathrm{mmol})$ was suspended in a hot solution prepared from water (30 ml) and $\mathrm{H}_{2} \mathrm{SO}_{4}(96 \%, 20 \mathrm{ml})$. The temperature was raised until all melem had dissolved. After cooling to $4{ }^{\circ} \mathrm{C}$ over night the colorless precipitate was collected by suction and dried on an absorptive piece of filter paper over night. Yield: $98 \mathrm{mg}$ (percentages are not given since the formula of the compound is uncertain as of now).

Compound B: Melem $(1.00 \mathrm{~g}, 4.587 \mathrm{mmol})$ was suspended in a hot solution prepared from water (35 ml) and $\mathrm{H}_{2} \mathrm{SO}_{4}(96 \%, 15 \mathrm{ml})$. The mixture was heated under reflux for approximately $10 \mathrm{~min}$. Melem did not completely dissolve. After cooling to $4{ }^{\circ} \mathrm{C}$ over night the colorless precipitate was collected by suction and dried on an absorptive piece of filter paper over night. Yield: $921 \mathrm{mg}$ (percentages are not given since the formula of the compound is uncertain as of now). 